\title{
A non-canonical unfolded protein response pathway and mitochondrial dynamics control the number of
}

\section{ER-mitochondria contact sites}

\author{
Rieko Kojima $^{1,2} \dagger$, Yuriko Kakimoto ${ }^{3, \dagger}$, Manatsu Shinmyo ${ }^{1}$, Kazuo Kurokawa ${ }^{4}$ \\ Akihiko Nakano ${ }^{4}$, Toshiya Endo ${ }^{5,6}$, Yasushi Tamura $^{1^{*}}$ \\ ${ }^{1}$ Faculty of Science, Yamagata University, 1-4-12 Kojirakawa-machi, Yamagata 990-8560, \\ Japan. \\ ${ }^{2}$ Toyama Prefectural Institute for Pharmaceutical Research, 17-1 Nakataikouyama, Imizu, \\ Toyama 939-0363, Japan \\ ${ }^{3}$ Department of Biochemistry and Molecular Biology, Graduate School of Medical Science, \\ Yamagata University, 2-2-2 lidanishi, Yamagata, 990-9585, Japan \\ ${ }^{4}$ Live Cell Super-Resolution Imaging Research Team, RIKEN Center for Advanced Photonics, \\ 2-1 Hirosawa, Wako, Saitama 351-0198, Japan. \\ ${ }^{5}$ Faculty of Life Sciences, Kyoto Sangyo University, Kamigamo-motoyama, Kita-ku, Kyoto \\ 603-8555, Japan. \\ ${ }^{6}$ Institute for Protein Dynamics, Kyoto Sangyo University, Kamigamo-motoyama, Kita-ku, Kyoto \\ 603-8555, Japan
}

tThese authors contributed equally to this work.

“Correspondence: tamura@sci.kj.yamagata-u.ac.jp 


\section{Abstract}

Mitochondria maintain their morphology and functions through the optimized balance between the mitochondrial fusion and division. Here we report a novel role of mitochondrial dynamics in controlling the number of ER-mitochondria encounter structure (ERMES) clusters in a yeast cell. Loss of mitochondrial fusion or division caused the increased or decreased number, respectively, of ERMES foci observed in cells. ERMES complexes, therefore, appear to cluster with each other and mitochondrial division may inhibit undesired ERMES hyper-clustering. Furthermore, our microscopic analyses suggest that ER stress induces dissociation of ERMES clusters, increasing the number of ERMES foci even in the absence of Ire1 and Hac1, which are essential factors for the UPR response. Interestingly, we found that ER stress leads to expansion of both the ER and mitochondrial membranes in an ERMES function-dependent manner. These findings imply that a cell is equipped with two independent regulatory mechanisms controlling the number of ER-mitochondria contact sites to meet the cellular as well as environmental demands. 


\section{Introduction}

Mitochondria are highly dynamic organelles and continuously fuse and divide to maintain their functionally optimized morphology. In yeast, two dynamin-related GTPases, Fzo1 and Mgm1 are known to mediate the mitochondrial outer and inner membrane (MOM and MIM) fusion, respectively [1]. Fzo1 is a MOM protein with two transmembrane (TM) segments and a large GTPase domain exposed to the cytosol. Mgm1 is present as two isoforms, long and short forms (1- and s-Mgm1). 1-Mgm1 is integrated into the MIM via its first N-terminal TM segment and exposes its GTPase domain to the intermembrane space (IMS). s-Mgm1 is a soluble IMS protein, which is released from the MIM after cleavage of the long form by a rhomboid protease Pcp1 located in the MIM [2-5]. Another multi-spanning MOM protein Ugo1 physically interacts with both Fzo1 in the MOM and Mgm1 in the MIM and likely couples the MOM and MIM fusion events [6,7]. Mitochondrial fusion is considered to contribute to attenuating mitochondrial oxidative damages, which could accumulate in mitochondria due to the generation of reactive oxygen species (ROS) as a byproduct of respiration. Impairment of the mitochondrial fusion leads to not only mitochondrial fragmentation by continuous mitochondrial fission but also to the loss of mitochondrial DNA (mtDNA). Consistent with the physiological importance of the mitochondrial fusion, mutations in Mfn2 and Opa1, mammalian Fzo1 and Mgm1, respectively, were found to cause human diseases such as Charcot-Marie-Tooth disease type 2A (CMT2A) and autosomal dominant optic atrophy (ADOA) [8]. Importantly, strong phenotypes associated with the cells defective in the mitochondrial fusion, growth defects as well as the loss of mtDNA can be suppressed by additional loss of division factors such as Dnm1 and Fis1 in yeast. Fragmented mitochondria in the cells with defective mitochondrial fusion are also restored to the ones in a tubular shape when mitochondrial division is additionally blocked [9-11].

Dnm1, another dynamin-related GTPase, is recruited to the MOM with the 
aid of receptor proteins such as Fis1, Mdv1, and Caf4 and assembled into a helical structure to split a mitochondrial tubule through its GTP hydrolysis-dependent conformational change [12-18]. Mitochondrial division in mammals further requires a classical dynamin, Dyn2, in addition to the mammalian counterpart of Dnm1, Drp1 (Lee et al., 2016). Mitochondrial division reportedly occurs at the mitochondria-ER contact sites, suggesting that the ERMES (ER-mitochondria encounter structure) complex, which directly tethers the ER to the MOM, may participate in mitochondrial division [19]. Mitochondrial tubules become constricted by physical association with the ER via the ERMES complex, and Dnm1 is targeted to such characteristic spots on mitochondria to mediate mitochondrial division [20].

The ERMES consists of four core subunits, ER-resident Mmm1, MOM-resident Mdm10 and Mdm34, and a peripheral membrane protein Mdm12 (Lang et al., 2015; Eisenberg-Bord et al., 2016; Murley and Nunnari, 2016; Tamura and Endo, 2017). These ERMES core subunits were initially identified as factors required for normal mitochondrial distribution and morphology [25-28]. Mitochondrial morphology is altered from a tubular to a spherical ball-like structure when one of these ERMES core components is absent, pointing out the close relationship of ERMES with mitochondrial morphogenesis. In addition to the role of ERMES in maintaining mitochondrial morphology, recent studies further revealed a function of ERMES in phospholipid transport between the ER and mitochondria (Kornmann et al., 2009; Kojima et al., 2016; Jeong et al., 2016, 2017; Kawano et al., 2018). These findings indicate that the ER-mitochondria tethering mediated by ERMES is vital for the functional integrity of mitochondria. Importantly, the ERMES complex constitutes large aggregates that can be observed as several discrete foci in a cell under a fluorescent microscope when an ERMES subunit is expressed as a GFP-fusion protein [25-28]. The number of ERMES in a cell should be precisely controlled to regulate the degree of mitochondrial division and phospholipid transfer. For example, the number of ERMES 
dots increases with a change in the metabolic state, such as a shift from the fermentation to non-fermentation culturing condition, which promotes mitochondrial proliferation [34]. Interestingly, physical association of mitochondria with the vacuole, termed vCLAMP (vacuole and mitochondria patch), exhibits an opposite behavior to ERMES with regard to the degree of membrane contacts; under non-fermentable conditions, Vps39, which is responsible for the formation of vCLAMP, is phosphorylated, resulting in a decreased number of vCLAMP [34]. These observations suggest that mitochondria-ER contacts and mitochondria-vacuole contacts mediated by ERMES and Vps39, respectively, are reciprocally regulated, and that the functions of ERMES and vCLAMP are partly overlapping [34,35]. A high-content imaging screen for yeast deletion mutants with an altered number of ERMES dots revealed that loss of Vps39 or that of mitochondrial division factors such as Dnm1 and Fis1 leads to an increased number of ERMES dots [35]. These previous observations suggest that spatially separated inter-organelle contacts are functionally connected, yet their underlying mechanism remains to be elucidated.

Here, we analyzed the relationship of mitochondrial fusion and division with the number of ERMES dots and found that loss of mitochondrial fusion or division caused increased or decreased number, respectively. Besides, we found that treatment of cells with tunicamycin or DTT, which induces ER stress, led to dissociation of the ERMES clusters, increasing the number of ERMES dots, independently of the mitochondrial division as well as unfolded protein response (UPR) components, Ire1 and Hac1. More importantly, we found that both the ER and mitochondrial membranes enlarge upon ER stress in a Mmm1-dependent manner probably for relieving the ER stress. These findings strongly suggest that dynamic changes in the number and size of ER-mitochondria contact sites play an important role in dealing with the stress conditions. 


\section{Results and Discussion}

\section{Mitochondrial fusion and division antagonistically affect the number of ERMES foci}

The main question we asked here is whether the cell has an active mechanism to regulate the number of inter-organelle contacts such as mitochondria-ER contact sites (MERCs), which are observed as dot-like structures under a microscope. If the cell is equipped with such a mechanism, we reasoned that regulators controlling the number of the MERCs could be localized in the MOM. To test this idea, we visualized MERCs with the split-GFP probes (Kakimoto et al., 2018) in yeast cells that lack each of the 53 different MOM proteins (Table S1). We noted that the number of MERCs significantly decreased in cells lacking Fis1, which functions as a receptor for mitochondrial division factor Dnm1 (Fig. S1) [14].

Since this observation is not consistent with the previous finding that the number of ERMES foci increased in the absence of Dnm1 or Fis1 [35], we further asked if the loss of mitochondrial division would affect the appearance of ERMES. We thus observed wild-type, dnml $1 \Delta$ and fis $1 \Delta$ cells expressing mitochondria-targeted RFP (Su9-RFP) and C-terminally GFP-tagged Mmm1 (Mmm1-GFP) under a confocal fluorescence microscope and acquired approximately $5-\mu \mathrm{m}$ z-stack images that cover a whole cell with a $0.2-\mu \mathrm{m}$ increment (Fig. 1). To minimize the undesired effects on the appearance of ERMES dots, arising from possible variations of the Mmm1-GFP expression level, we adopted the stable expression of Mmm1-GFP from the chromosome. Maximum projection images reconstituted from the z-stacks showed that ERMES dots were localized on mitochondrial tubules in wild-type, $d n m 1 \Delta$, and fis $1 \Delta$ cells although mitochondrial distribution was altered due to lack of mitochondrial division in dnm $1 \Delta$ and fis $1 \Delta$ cells; wild-type, dnml $\Delta$, and fis $1 \Delta$ cells contained 5.8, 3.5 and 3.0 ERMES dots per cell on average, respectively. These results indicate that loss of mitochondrial division leads to a decrease in the number of ERMES dots. The 
reduced ERMES number observed in mitochondrial division-deficient cells was not due to decreased levels of ERMES subunits since the protein levels of Mmm1, Mdm12 and Mdm34 were all comparable between $d n m 1 \Delta$ cells and wild-type cells (Fig. S2A).

We then re-examined the previously reported effects of loss of vCLAMP components on the number of ERMES foci. Although the vCLAMP component Vps39 was found as a factor whose absence led to an increase in the number of ERMES foci [35], we did not observe a drastic change in the number of ERMES foci in vps394 cells as compared with that of wild-type cells (Fig. 1A, B). On the other hand, loss of another vCLAMP component Ypt7 led to a slight increase in the ERMES dot number. These results collectively suggest that factors other than vCLAMP, e.g., the growth phase, growth media, or yeast strain backgrounds could also affect the appearance of ERMES dots in a complex manner.

We next estimated the number of Mmm1-GFP molecules included in a single ERMES dot from their GFP signals using Cse4-GFP as a standard [36,37]. Interestingly, we found that each of the ERMES dots in $d n m 1 \Delta$ and fis $1 \Delta$ cells contains 335 and 351 of Mmm1-GFP molecules on average, respectively, which are significantly larger than 196 molecules per dot in wild-type cells (Fig. 1C). The average numbers of Mmm1-GFP molecules per ERMES dot were similar in vps39 4 and slightly smaller in ypt7 $\Delta$ cells as compared with that in wild-type cells. These results suggest that the reduced number of ERMES dots in yeast mutant cells defective in mitochondrial division reflects enhanced clustering of the preexisting ERMES clusters. The proper mitochondrial division may thus inhibit unnecessary clustering of ERMES complexes, thereby maintaining the appropriate number of the MERCs in cells.

If mitochondrial division suppresses hyper-clustering of preexisting ERMES clusters and thereby controls the optimum number of ERMES foci, loss of mitochondrial fusion will, in turn, increase the number of ERMES foci due to ongoing mitochondrial division. We thus deleted mitochondrial fusion genes, FZO1, UGO1 or 
MGM1 and observed ERMES dots visualized with Mmm1-GFP. Since the lack of mitochondrial fusion causes loss of mtDNA, we used a $r h o^{0}$ strain, in which mitochondrial DNA lacks, as a control. Supporting our above idea, defects in mitochondrial fusion by deletion of the genes for the MOM fusion, FZO1, and UGO1, increased the number of ERMES foci while the loss of mtDNA alone did not (Fig. 1A, B). Deletion of the MIM fusion gene, $M G M 1$, caused a marginal increase in the ERMES dot number, which could reflect the fact that Mgm1-mediated MIM fusion is not entirely coupled with the Fzo1-mediated MOM fusion [38]. Further supporting the role of mitochondrial division in controlling the number of ERMES foci, loss of the division gene DNMI together with the loss of a fusion gene, FZO1, UGO1, or MGMI reversed the phenotypes. That is, fzol $\Delta d n m 1 \Delta$, ugol $\Delta d n m 1 \Delta$, and mgml $1 \Delta d n m 1 \Delta$ cells exhibited tubular mitochondria due to simultaneous defects in mitochondrial fusion and division as reported, and the number of ERMES foci was changed to the level similar to the one for $d n m 1 \Delta$ cells, regardless of the simultaneous deletion of FZO1, UGO1 or MGM1 (Fig. 1A, B). Besides, the estimated number of Mmm1-GFP molecules in an ERMES dot was clearly smaller when mitochondrial fusion was inhibited than those of wild-type and $r h o^{0}$ cells while the Mmm1-GFP particles per ERMES dot drastically increased to the level similar to dnml $\triangle$ cells when DNM1 was deleted together with FZO1, UGO1 or MGM1 (Fig. 1C). These results suggest that mitochondrial fusion and division antagonistically affect the number of ERMES foci by controlling the clustering of preexisting ERMES foci.

\section{Preexisting ERMES foci cluster together}

To directly test the idea that the preexisting ERMES foci gather together, we utilized a yeast mating assay with which we can monitor mitochondrial fusion directly (Sesaki et al., 2003b) (Fig. 2A). Briefly, we constructed two types of yeast cells with the opposing mating types (MATa and MAT $\alpha$ ) that express Mmm1-GFP or Mmm1-mScarlet 
under the control of the GALI promoter. Then we examined how the ERMES foci behave after cell fusion. If the preexisting ERMES foci tend to cluster together, the green- and red-labeled foci should be merged or adjacent to each other after cell fusion (Fig. 2A). To exclude the mere possibility that newly synthesized Mmm1-GFP congregates into preexisting ERMES foci consisting of Mmm1-mScarlet, or vice versa, after cell fusion, we suppressed the expression of Mmm1-GFP and Mmm1-mScarlet before mating by cultivating the cells in a glucose-containing medium YPD for 4 hours. Immunoblotting of whole cell lysates confirmed that Mmm1-GFP and Mmm1-mScarlet expression was shut off after cultivation in YPD (Fig. 2B). Strikingly, most ERMES foci labeled with different fluorescent proteins were adjacent to each other rather than completely merged (Fig. 2C). This observation indicates that the different preexisting ERMES foci do not completely fuse, but instead contact with each other to form large clusters. We thus propose that the proper balance of mitochondrial fusion and division regulates clustering of preexisting ERMES foci, thereby maintaining the optimum number of ERMES foci (Fig. 2D).

\section{The ERMES foci number increases upon ER stress in an Ire1- and Hac1-independent manner}

Studies using mammalian cultured cells suggested that the MERC number increased under ER stress conditions [39-41]. In contrast, a recent study reported that ER stress did not drastically affect MERC foci visualized with split-GFP probes in U2OS cells [42]. We thus treated yeast cells expressing Mmm1-GFP with tunicamycin or DTT for 2 hours to induce ER stress, and then observed ERMES foci under a fluorescence microscope. Strikingly, the number of ERMES foci increased about 2-fold upon treatment with tunicamycin or DTT (Fig. 3A, C). We confirmed that tunicamycin treatment does not affect the amounts of ERMES components such as Mmm1 and Mdm12 although it decreased the level of N-glycosylated Mmm1 and Mmm1-GFP (Fig. 
S2B). Therefore, the sharp increase in the number of ERMES foci was not due to increased expression of ERMES components. The growth in the ERMES dot number upon ER stress was not due to accelerated mitochondrial division, either, since tunicamycin or DTT treatment caused an increase in the number of ERMES foci even in the absence of Dnm1 (Fig. 3B, C). Another possible explanation for the increased number of ERMES foci is that the ERMES clusters dissociate upon ER stress. We examined this possibility by super-resolution confocal live imaging microscopy (SCLIM), which enable us to obtain time-lapse fluorescent images of whole yeast cells at super-high resolution $[43,44]$. Interestingly, we could observe splits of the ERMES dots after the tunicamycin treatment (Fig. S2C). Consistently, we confirmed that the estimated number of Mmm1-GFP molecules present in an ERMES dot after the tunicamycin or DTT treatment is smaller than that of nontreated wild-type cells (Fig. 3D). These findings strongly suggest that dissociation of ERMES clusters is at least partly responsible for the increased ERMES foci number.

We next asked whether the increase in the number of ERMES foci would depend on the unfolded protein response (UPR) [45]. In yeast, an ER-resident type I TM protein Ire1 senses accumulation of aberrant proteins in the ER. Then Ire1 undergoes self-oligomerization, which triggers both kinase and endonuclease activation of Ire1, hence leading to Ire1-mediated HAC1 mRNA splicing [46]. The spliced mature HACl mRNAs produce the functional form of the Hac1 transcription factor, which activates a number of genes repressed under non-stress conditions [47,48]. We thus asked if a loss of either Ire1 or Hac1 would affect the increase in the number of ERMES foci under ER stress conditions. Surprisingly, neither loss of Ire1 nor Hac1 suppressed the increase in the number of ERMES foci (Fig. 3B, F), suggesting that a non-canonical mechanism independent of the IRE1/HAC mediates the ER-stress dependent change in the ERMES foci number. What mechanism underlies the dissociation of ERMES clusters under ER stress conditions? One possibility is that ER 
stress leads to dissociation of ERMES clusters through modifications like phosphorylation and ubiquitination of the ERMES components. However, this is unlikely because we did not observe band shifts of any of the ERMES components by SDS-PAGE followed by immunoblotting (Fig. S2C). We also confirmed that yeast cells expressing Mdm34-3PA mutant, which lacks the PY motif critical for its ubiquitination by Rsp5 E3 ubiquitin ligase, showed a similar increase in the ERMES dot number after tunicamycin and DTT treatments (Fig. S2D). Another possibility is that the ER stress induces alteration of phospholipid compositions of the ER membrane and/or MOM, which may affect the conformation and/or assembly of the ERMES complex, resulting in the split of the ERMES foci. However, we confirmed that at least phospholipid class compositions were not altered by tunicamycin and DTT treatments (data not shown).

\section{ER-stress leads to expansion of both the ER and mitochondria membranes through ERMES functions}

What is the possible physiological role of the ER stress-triggered increase in the ERMES dot number? A previous study showed that the ER membranes significantly expand upon ER stress in an Ino2/Ino4-dependent manner to attenuate the ER stress [49]. Ino2/Ino4 transcription factors are known to activate transcriptions of a series of genes for phospholipid synthases [50]. On the other hand, it is well known that phospholipids have to shuttle between the ER and mitochondria via ERMES for their proper syntheses [51-54]. Therefore, it is attractive to assume that the combination of transcriptional activation of the genes for phospholipid synthetic enzymes and the increase in the number of ERMES dots, which represent phospholipid transport sites between the ER and mitochondria, cooperatively enhance phospholipid biosynthesis under ER stress conditions. Supporting this idea, we found that the ER stress-dependent ER membrane expansion is partly suppressed by loss of Mmm1 (Fig. 4A). $\quad$ 90\% of 
wild-type cells showed the ER structure with elongated membranes after ER stress inductions whereas $\sim 50 \%$ of $m m m 1 \Delta$ cells contained such a developed ER structure (Fig. 4C). More importantly, we found that tunicamycin and DTT treatments led to a significant increase in mitochondrial structure as well (Fig. 4B, D). We noticed that the loss of Mmm1 abolished the development of mitochondria membranes under these ER stress conditions. Similar mitochondrial expansion was observed when mitochondrial division factor Dnm1 is absent. It is known that the lack of a division factor Dnm1 leads to a change in mitochondrial morphology from tubular structures to net-like structures $[9,10]$. The net-like mitochondrial shape was further enhanced likely owing to the mitochondrial membrane expansion by the ER stress (Fig. 3A, F). There results suggest that expansion of the ER and mitochondria membranes requires normal ERMES functions. Consistent with this idea, we found that mmml-1 cells became more susceptible to the tunicamycin treatment as compared with the corresponding wild-type strain (Fig, 4E). Collectively, our results strongly suggest a previously overlooked fact that ER stress induces the Ire1/Hac1-independent dissociation of ERMES clusters, which could activate phospholipid biogenesis in cooperation with the Ire1/Hac1-dependent transcriptional upregulation of phospholipid synthase genes. The enhanced phospholipid biogenesis would be critical for expansion of not only the ER but also mitochondrial membranes to buffer the ER stress (Fig. 4F).

However, there are some unresolved issues here. For example, it is still unclear how Ino2/Ino4 transcription factors are activated in Ire1/Hac1-dependent manner upon ER stress. A previous study suggested a possibility that Hac1 inhibits the function of Opi1 as a repressor for Ino2/Ino4 although this has not yet been revealed (Fig. 4F). Whether the lipid flux between the ER and mitochondria is really activated under ER stress conditions is also an important question to be assessed. Besides, what triggers the dissociation of ERMES clusters remains unclear. Although we confirmed that ER stress induction does not alter compositions of at least head group of 
phospholipids, it is still possible that acyl-chain variations cause conformational changes in the ERMES clusters, which could lead to their dissociation. Alternatively, protein modifications or additional binding partner for ERMES subunits may cause the dissociation of ERMES clusters under ER stress conditions. Detail mechanisms underlying the dynamic feature of the number of ERMES clusters should be investigated in future studies

\section{Material and Methods}

\section{Strains, plasmids, primers, and growth conditions}

In this study, we used a Saccharomyces cerevisiae strain, FY833 (MATa ura3-52

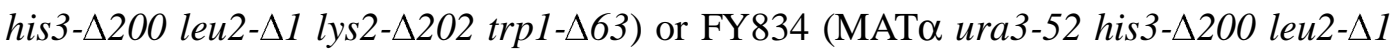
lys $2-\Delta 202$ trp1- $\triangle 63)$ as background strains [55]. All the yeast cells used in this study are listed in Table S1. Yeast cells expressing Mmm1-GFP originated from FY833 was used as a wild-type strain throughout this study $[55,56]$. The C-terminal GFP or mScarlet tagging, the introduction of the GALl promoter in front of the $M M M 1$ gene and gene disruptions were performed by homologous recombination using the appropriate gene cassettes amplified from plasmids listed in Table S2 [57,58]. A pair of primers, \#YU1116/1117, \#NU831/832, \#NU829/830, \#YU29/30, \#YU31/32, \#YU33/34, \#YU1347/1348, or \#YU1349/1350 was used to amplify the gene cassette for the disruption for FIS1, VPS39, YPT7, FZO1, UGO1, MGM1, IRE1 or HAC1 gene, respectively. For the introduction of GFP or Scarlet tag for MMM1 and CSE4, the GAL1 promoter for $M M M 1$ or 3PA-GFP tag for $M D M 34$, the appropriate gene cassettes were amplified with a pair of primer \#NU1174/1175, \#YU1518/1519, \#YU1563/1564, or \#NU1071/1072, respectively. Yeast cells were grown in YPD (1\% yeast extract, 2\% polypeptone, and $2 \%$ glucose $)$, SCD $(0.67 \%$ yeast nitrogen base without amino acids, $0.5 \%$ casamino acid, and $2 \%$ glucose $)$, SD (0.67\% yeast nitrogen base without amino acids, $0.13 \%$ drop-out amino acid mix and $2 \%$ glucose) media with appropriate 
supplements. The drop-out amino acid mix was a mixture of $2.6 \mathrm{~g}$ adenine, $6.0 \mathrm{~g}$ L-aspartic acid, $12 \mathrm{~g}$ L-threonine, $2.6 \mathrm{~g}$ L-asparagine, $1.8 \mathrm{~g}$ L-tyrosine, $6.0 \mathrm{~g}$ L-glutamic acid, 2.6 g L-glutamine, $2.6 \mathrm{~g}$ glycine , $2.6 \mathrm{~g}$ L-alanine, $2.6 \mathrm{~g}$ L-isoleucine, $1.2 \mathrm{~g}$ L-methionine, 3.0 g L-phenylalanine, 2.6 g L-proline, $22.6 \mathrm{~g}$ L-serine, $9.0 \mathrm{~g}$ L-valine and $2.6 \mathrm{~g}$ L-cysteine.

\section{Fluorescence microscopy}

Logarithmically growing yeast cells cultivated in SCD or SD media were observed under Olympus IX83 microscope with a CSU-X1 confocal unit (Yokogawa), a 100 x, 1.4 NA, objective (UPlanSApo, Olympus) and an sCMOS camera (Zyla 5.5; Andor) manipulated by MetaMorph software (Molecular Devices). When observed mScarlet signal, we used an EM-CCD camera (Evolve 512; Photometrics). GFP or $\mathrm{RFP} / \mathrm{mScarlet}$ were excited by $488-\mathrm{nm}$ or $561-\mathrm{nm}$ laser (OBIS, Coherent) and the emission was passed through 520/35-nm or 617/73-nm band-pass filter, respectively. The confocal fluorescent sections were collected every $0.2 \mu \mathrm{m}$ from the upper to the bottom surface of yeast cells. The obtained confocal images were subjected to maximum projection using Image $\mathrm{J}$ software. For counting ERMES dots, Mmm1-GFP dots were automatically picked by TransFluor, a macro for MetaMorph software, using the "Pits" algorithm. The resulting images containing picked dots were then subjected for counting. Signal intensities of ERMES and Cse4-GFP dots were calculated by using Image J software.

SCLIM was developed by combining Olympus model IX-71 inverted fluorescence microscope with a UPlanSApo 100 X NA 1.4 oil objective lens (Olympus, Japan), a high-speed and high-signal-to noise-ratio spinning-disk confocal scanner (Yokogawa Electric, Japan), a custom-made spectroscopic unit, image intensifiers (Hamamatsu Photonics, Japan) equipped with a custom-made cooling system, magnification lens system for giving $266.7 \mathrm{X}$ final magnification, and three EM-CCD cameras 
(Hamamatsu Photonics, Japan) for green, red, and infrared observation [43]. Image acquisition was executed by custom-made software (Yokogawa Electric, Japan). For 3D time-lapse imaging, we collected optical sections spaced $0.2 \mu \mathrm{m}$ apart in stacks by oscillating the objective lens vertically with a custom-made piezo actuator. $\mathrm{Z}$ stack images were converted to 3D voxel data and processed by deconvolution with Volocity (Perkin Elmer, MA) using the theoretical point-spread function for spinning-disk confocal microscopy.

\section{Immunoblotting and antibodies}

For immunoblotting, proteins transferred to PVDF membranes (Immobilon-FL or Immobilon-P, Millipore) were detected by fluorophore- or HRP-conjugated to secondary antibodies (Cy5 AffiniPure Goat Anti-Rabbit IgG (H+L) from Jackson ImmunoResearch Labs or Goat anti-Rabbit IgG (H+L) Cross-Adsorbed Secondary Antibody, HRP from Thermo Fisher Scientific) and analyzed with a Typhoon imager (GE Healthcare) or LAS-4000 mini (Fujifilm).

\section{Statistical analyses}

The number of ERMES per a cell and signal intensities of ERMES and Cse4-GFP dots were shown as box and whisker plots (Tukey). The sample number was shown in figure legends. Data are shown as means with SEM or SD as indicated in the figure legends. The Student's t-test with Welch's correction were performed for the statistical analyses using Prism 6 (GraphPad).

\section{Acknowledgments}

We thank K. Shishido, M. Hashimoto, and T. Sasaki for their great technical assistance and Profs. Robert E. Jensen and Hiromi Sesaki for mmml-1 and its corresponding wild-type strain. We are grateful to the members of the Tamura and Endo laboratories for helpful discussion. This work was supported by JSPS KAKENHI (Grant Numbers 
17H06414 and 19H03174 to YT, 17H06413 to KK and AN, 17H06420 and 18H05275 to K.K, and $15 \mathrm{H} 05705$ and 22227003 to TE), AMED-PRIME (Grant Number JP19gm5910026) from Japan Agency for Medical Research and Development, AMED (YT), and a CREST Grant (JPMJCR12M1) from JST (TE).

\section{Author Contributions}

TE, RK, and YT designed the study. RK performed the experiments shown in Fig. 1 and 2. YK performed the analysis shown in Fig. 4. SM screened for MOM proteins whose loss affected the appearance of the ER-mitochondria contact sites visualized with split-GFP probes. KK and AN helped to conduct live-cell imaging with SCLIM and edited the manuscript. TE and YT wrote the paper.

\section{Conflict of interest}

The authors declare that they have no conflict of interest.

\section{References}

1. Tamura Y, Itoh K, Sesaki H (2011) SnapShot: Mitochondrial dynamics. Cell 145:.

2. Sesaki H, Southard SM, Yaffe MP, Jensen RE (2003) Mgm1p, a Dynamin-related GTPase, Is Essential for Fusion of the Mitochondrial Outer Membrane. Mol Biol Cell 14: 2342-2356.

3. Wong ED, Wagner JA, Gorsich SW, McCaffery JM, Shaw JM, Nunnari J (2000) The dynamin-related GTPase, Mgm1p, is an intermembrane space protein required for maintenance of fusion competent mitochondria. J Cell Biol 151: 341-352.

4. Sesaki H, Southard SM, Aiken Hobbs AE, Jensen RE (2003) Cells lacking Pcp1p/Ugo2p, a rhomboid-like protease required for Mgm1p processing, lose mtDNA and mitochondrial structure in a Dnm1p-dependent manner, but remain competent for mitochondrial fusion. Biochem Biophys Res Commun 308: 276-283. 
5. McQuibban GA, Saurya S, Freeman M (2003) Mitochondrial membrane remodelling regulated by a conserved rhomboid protease. Nature 423: 537-541.

6. Sesaki H, Jensen RE (2004) Ugo1p links the Fzo1p and Mgm1p GTPases for mitochondrial fusion. J Biol Chem 279: 28298-28303.

7. Wong ED, Wagner JA, Scott S V., Okreglak V, Holewinske TJ, Cassidy-Stone A, Nunnari J (2003) The intramitochondrial dynamin-related GTPase, Mgm1p, is a component of a protein complex that mediates mitochondrial fusion. $J$ Cell Biol 160: 303-311.

8. Itoh K, Nakamura K, Iijima M, Sesaki H (2013) Mitochondrial dynamics in neurodegeneration. Trends Cell Biol 23: 64-71.

9. Sesaki H, Jensen RE (1999) Division versus fusion: Dnm1p and Fzo1p antagonistically regulate mitochondrial shape. J Cell Biol 147: 699-706.

10. Bleazard W, McCaffery JM, King EJ, Bale S, Mozdy A, Tieu Q, Nunnari J, Shaw JM (1999) The dynamin-related GTPase Dnm1 regulates mitochondrial fission in yeast. Nat Cell Biol 1: 298-304.

11. Kojima R, Kakimoto Y, Furuta S, Itoh K, Sesaki H, Endo T, Tamura Y (2019) Maintenance of Cardiolipin and Crista Structure Requires Cooperative Functions of Mitochondrial Dynamics and Phospholipid Transport. Cell Rep 26: 518-528.e6.

12. Cerveny KL, McCaffery JM, Jensen RE (2001) Division of Mitochondria Requires a Novel DNM1 -interacting Protein, Net2p. Mol Biol Cell 12: 309-321.

13. Tieu Q, Nunnari J (2000) Mdv1p Is a Wd Repeat Protein That Interacts with the Dynamin-Related Gtpase, Dnm1p, to Trigger Mitochondrial Division. J Cell Biol 151: 353-366.

14. Mozdy AD, McCaffery JM, Shaw JM (2000) Dnm1p GTPase-mediated mitochondrial fission is a multi-step process requiring the novel integral membrane component Fis1p. J Cell Biol 151: 367-379.

15. Cerveny KL, Jensen RE (2003) The WD-repeats of Net2p Interact with Dnm1p and Fis1p to Regulate Division of Mitochondria. Mol Biol Cell 14: 4126-4139.

16. Griffin EE, Graumann J, Chan DC (2005) The WD40 protein Caf4p is a component of the mitochondrial fission machinery and recruits Dnm1p to mitochondria. J Cell Biol 170: 237-248. 
17. Lackner LL, Horner JS, Nunnari J (2009) Mechanistic Analysis of a Dynamin Effector. Science (80- ) 325: 874-877.

18. Mears JA, Lackner LL, Fang S, Ingerman E, Nunnari J, Hinshaw JE (2011) Conformational changes in Dnm1 support a contractile mechanism for mitochondrial fission. Nat Struct Mol Biol 18: 20-26.

19. Friedman JR, Lackner LL, West M, DiBenedetto JR, Nunnari J, Voeltz GK (2011) ER Tubules Mark Sites of Mitochondrial Division. Science (80- ) 334: $358-362$.

20. Murley A, Lackner LL, Osman C, West M, Voeltz GK, Walter P, Nunnari J (2013) ER-associated mitochondrial division links the distribution of mitochondria and mitochondrial DNA in yeast. Elife 2: e00422.

21. Lang A, John Peter AT, Kornmann B (2015) ER-mitochondria contact sites in yeast: beyond the myths of ERMES. Curr Opin Cell Biol 35: 7-12.

22. Eisenberg-Bord M, Shai N, Schuldiner M, Bohnert M (2016) A Tether Is a Tether Is a Tether: Tethering at Membrane Contact Sites. Dev Cell 39: 395-409.

23. Tamura Y, Endo T (2017) Role of intra- and inter-mitochondrial membrane contact sites in yeast phospholipid biogenesis. In, Advances in Experimental Medicine and Biology pp 121-133.

24. Murley A, Nunnari J (2016) The Emerging Network of Mitochondria-Organelle Contacts. Mol Cell 61: 648-653.

25. Burgess SM, Delannoy M, Jensen RE (1994) MMM1 encodes a mitochondrial outer membrane protein essential for establishing and maintaining the structure of yeast mitochondria. J Cell Biol 126: 1375-1391.

26. Sogo LF, Yaffe MP (1994) Regulation of mitochondrial morphology and inheritance by Mdm10p, a protein of the mitochondrial outer membrane. J Cell Biol 126: 1361-1373.

27. Berger KH, Sogo LF, Yaffe MP (1997) Mdm12p, a component required for mitochondrial inheritance that is conserved between budding and fission yeast. $J$ Cell Biol 136: 545-553.

28. Dimmer KS, Fritz S, Fuchs F, Messerschmitt M, Weinbach N, Neupert W, Westermann B (2002) Genetic basis of mitochondrial function and morphology in Saccharomyces cerevisiae. Mol Biol Cell 13: 847-853. 
29. Kornmann B, Currie E, Collins SR, Schuldiner M, Nunnari J, Weissman JS, Walter P (2009) An ER-mitochondria tethering complex revealed by a synthetic biology screen. Science 325: 477-481.

30. Kojima R, Endo T, Tamura Y (2016) A phospholipid transfer function of ER-mitochondria encounter structure revealed in vitro. Sci Rep 6: 30777.

31. Jeong H, Park J, Lee C (2016) Crystal structure of Mdm12 reveals the architecture and dynamic organization of the ERMES complex. EMBO Rep 17: $1857-1871$.

32. Jeong H, Park J, Jun Y, Lee C (2017) Crystal structures of Mmm1 and Mdm12-Mmm1 reveal mechanistic insight into phospholipid trafficking at ER-mitochondria contact sites. Proc Natl Acad Sci 114: E9502-E9511.

33. Kawano S, Tamura Y, Kojima R, Bala S, Asai E, Michel AH, Kornmann B, Riezman I, Riezman H, Sakae Y, et al. (2018) Structure-function insights into direct lipid transfer between membranes by Mmm1-Mdm12 of ERMES. J Cell Biol 217: 959-974.

34. Hönscher C, Mari M, Auffarth K, Bohnert M, Griffith J, Geerts W, van der Laan M, Cabrera M, Reggiori F, Ungermann C (2014) Cellular metabolism regulates contact sites between vacuoles and mitochondria. Dev Cell 30: 86-94.

35. Elbaz-Alon Y, Rosenfeld-Gur E, Shinder V, Futerman AH, Geiger T, Schuldiner M (2014) A Dynamic Interface between Vacuoles and Mitochondria in Yeast. Dev Cell 30: 95-102.

36. Lawrimore J, Bloom KS, Salmon ED (2011) Point centromeres contain more than a single centromere-specific Cse4 (CENP-A) nucleosome. J Cell Biol 195: $573-582$.

37. Yamamoto H, Kakuta S, Watanabe TM, Kitamura A, Sekito T, Chika K-K, Ichikawa R, Kinjo M, Ohsumi Y (2012) Atg9 vesicles are an important membrane source during early steps of autophagosome formation. J Cell Biol 198: $219-233$.

38. Meeusen S (2004) Mitochondrial Fusion Intermediates Revealed in Vitro. Science (80- ) 305: 1747-1752.

39. Bravo R, Vicencio JM, Parra V, Troncoso R, Munoz JP, Bui M, Quiroga C, Rodriguez AE, Verdejo HE, Ferreira J, et al. (2011) Increased ER-mitochondrial 
coupling promotes mitochondrial respiration and bioenergetics during early phases of ER stress. J Cell Sci 124: 2511-2511.

40. Zhao YG, Chen Y, Miao G, Zhao H, Qu W, Li D, Wang Z, Liu N, Li L, Chen S, et al. (2017) The ER-Localized Transmembrane Protein EPG-3/VMP1 Regulates SERCA Activity to Control ER-Isolation Membrane Contacts for Autophagosome Formation. Mol Cell 67: 974-989.e6.

41. Cieri D, Vicario M, Giacomello M, Vallese F, Filadi R, Wagner T, Pozzan T, Pizzo P, Scorrano L, Brini M, et al. (2018) SPLICS: a split green fluorescent protein-based contact site sensor for narrow and wide heterotypic organelle juxtaposition. Cell Death Differ 25: 1131-1145.

42. Yang Z, Zhao X, Xu J, Shang W, Tong C (2018) A novel fluorescent reporter detects plastic remodeling of mitochondria-ER contact sites. J Cell Sci 131:

43. Kurokawa K, Ishii M, Suda Y, Ichihara A, Nakano A (2013) Live cell visualization of golgi membrane dynamics by super-resolution confocal live imaging microscopy. Elsevier Inc.

44. Kurokawa K, Okamoto M, Nakano A (2014) Contact of cis-Golgi with ER exit sites executes cargo capture and delivery from the ER. Nat Commun 5: 3653.

45. Walter P, Ron D (2011) The Unfolded Protein Response: From Stress Pathway to Homeostatic Regulation. Science (80- ) 334: 1081-1086.

46. Korennykh A V., Egea PF, Korostelev AA, Finer-Moore J, Zhang C, Shokat KM, Stroud RM, Walter P (2009) The unfolded protein response signals through high-order assembly of Ire1. Nature 457: 687-693.

47. Sidrauski C, Walter P (1997) The transmembrane kinase Ire1p is a site-specific endonuclease that initiates mRNA splicing in the unfolded protein response. Cell 90: 1031-1039.

48. Kawahara T, Yanagi H, Yura T, Mori K (1997) Endoplasmic Reticulum Stress-induced mRNA Splicing Permits Synthesis of Transcription Factor Hac1p/Ern4p That Activates the Unfolded Protein Response. Mol Biol Cell 8: 1845-1862.

49. Schuck S, Prinz WA, Thorn KS, Voss C, Walter P (2009) Membrane expansion alleviates endoplasmic reticulum stress independently of the unfolded protein response. J Cell Biol 187: 525-536. 
50. Henry SA, Kohlwein SD, Carman GM (2012) Metabolism and Regulation of Glycerolipids in the Yeast Saccharomyces cerevisiae. Genetics 190: 317-349.

51. Tamura Y, Sesaki H, Endo T (2014) Phospholipid Transport via Mitochondria. Traffic 15: 933-945.

52. Tatsuta T, Scharwey M, Langer T (2014) Mitochondrial lipid trafficking. Trends Cell Biol 24: 44-52.

53. Dimmer KS, Rapaport D (2017) Mitochondrial contact sites as platforms for phospholipid exchange. Biochim Biophys Acta - Mol Cell Biol Lipids 1862: 69-80.

54. Tamura Y, Kawano S, Endo T (2019) Organelle contact zones as sites for lipid transfer. J Biochem 165: 115-123.

55. Winston F, Dollard C, Ricupero-Hovasse SL (1995) Construction of a set of convenient Saccharomyces cerevisiae strains that are isogenic to S288C. Yeast 11: 53-55.

56. Tamura Y, Onguka O, Hobbs AEA, Jensen RE, Iijima M, Claypool SM, Sesaki H (2012) Role for Two Conserved Intermembrane Space Proteins, Ups1p and Up2p, in Intra-mitochondrial Phospholipid Trafficking. J Biol Chem 287: 15205-15218.

57. Longtine MS, Mckenzie III A, Demarini DJ, Shah NG, Wach A, Brachat A, Philippsen P, Pringle JR (1998) Additional modules for versatile and economical PCR-based gene deletion and modification in Saccharomyces cerevisiae. Yeast 14: 953-961.

58. Kakimoto Y, Tashiro S, Kojima R, Morozumi Y, Endo T, Tamura Y (2018) Visualizing multiple inter-organelle contact sites using the organelle-targeted split-GFP system. Sci Rep 8: 6175.

Figure 1. Mitochondrial fusion and division antagonistically regulate the number of ERMES dots.

A The indicated yeast cells expressing Mmm1-GFP and mitochondria-targeted RFP (Su9-RFP) were imaged under a confocal fluorescence microscope. Maximum projection images were shown. Scale bars, $5 \mu \mathrm{m}$. 
B Box and whisker plots show the distribution of the number of ERMES dots per a cell. SD means standard deviation. $* *: p<0.001, * * * *: p<0.0001, p$ values were obtained from the unpaired two-tailed t-test with Welch's correction.

C Quantifications of signal intensities of ERMES dots in the indicated cells using Cse4-GFP signal as a standard. Box and whisker plots show the estimated number of Mmm1-GFP molecules included in a single ERMES dot. The average intensity of Cse4-GFP foci was set to 80 U. ns: not significant, **: $p<0.001$, **** $: p<$ $0.0001, p$ values were obtained from the unpaired two-tailed t-test with Welch's correction.

Figure 2. Pre-existing ERMES dots cluster together upon cell fusion.

A A schematic diagram of the mating assay showing possible patterns of the ERMES dots. (1) The preexisting ERMES foci do not cluster together. $(2,3)$ The preexisting ERMES foci merge completely (2) or contact each other (3).

B Whole cell extracts prepared from yeast cells after shutting off the expression of Mmm1-GFP or Mmm1-mScarlet were subjected to immunoblotting using antibodies against Mmm1 and Tim23.

C Yeast zygotes obtained by mating haploid cells containing ERMES dots labeled with different fluorescent colors. Scale bars, $5 \mu \mathrm{m}$.

D A working model for regulating the number of ERMES dots by mitochondrial fusion and division.

Figure 3. The number of ERMES dots significantly increases upon treatments with ER stress inducers independently of mitochondrial division and the conventional UPR pathway.

A Wild-type (WT) and dnml cells expressing Mmm1-GFP and mitochondria-targeted RFP (Su9-RFP) were incubated in SCD media with or 
without $1 \mu \mathrm{g} / \mathrm{ml}$ tunicamycin $(+\mathrm{Tm})$ or $3 \mathrm{mM}$ DTT $(+\mathrm{DTT})$ for $2 \mathrm{~h}$ and then observed under a confocal fluorescence microscope. Maximum projection images reconstituted from z-stacks were shown. Scale bars, $5 \mu \mathrm{m}$.

B irel $\Delta$ and hacl $\Delta$ cells expressing Mmm1-GFP and mitochondria-targeted RFP (Su9-RFP) were observed under a confocal fluorescence microscope as in A.

C Box and whisker plots show the number of ERMES dots per a wild-type or a $d n m 1 \Delta$ cell with or without the treatment inducing ER stress. Numbers indicated above the plots show mean. $\mathrm{n}=199,194$ and $132(\mathrm{WT}$, None, Tm and DTT) and $\mathrm{n}$ $=179,174$ and $105(d n m 1 \Delta$, None, Tm and DTT). $* * * *: p<0.0001, p$ values were obtained from the unpaired two-tailed t-test with Welch's correction.

D Quantifications of signal intensities of ERMES dots in wild-type cells with or without the DTT or Tm treatment as in Fig. 1C. **: $p=0.0019$, **** $: p<0.0001$, $p$ values were obtained from the unpaired two-tailed t-test with Welch's correction.

E The number of ERMES dots per an irel $\Delta$ or a hacl $\Delta$ cell with or without the treatment inducing ER stress was quantified as in Fig. 4C. $n=386,252$ and 315 (irel $\Delta$, None, + Tm and $+\mathrm{DTT})$ and $\mathrm{n}=386,323$ and 300 (hacl $\Delta$, None, +Tm and +DTT). ${ }^{* * * *}: p<0.0001, p$ values were obtained from the unpaired two-tailed t-test with Welch's correction.

F Quantifications of the ratio of cells containing large net-like (Net), tubular (T), and the other shaped mitochondria in Fig. 3B. Total 436, 451 and 245 cells for None (without inducing ER-stress), +Tm (with tunicamycin treatment) or +DTT (with DTT treatment), respectively, were examined in three independent experiments. Values are mean \pm S.E. $(n=3)$

Figure 4. ER stress causes membrane expansion of both the ER and mitochondria in a Mmm1-dependent manner.

A Wild-type and $m m m l \Delta$ cells expressing the ER-targeted or GFP were imaged under 
a confocal fluorescence microscope. Maximum projection images were shown. Scale bars, $5 \mu \mathrm{m}$. "e" in (A) represents the expanded ER structure.

B Wild-type and mmml $\Delta$ cells expressing the mitochondria-targeted GFP were imaged as in A.

C Ratio of cells containing the expanded ER membranes. A Total of over 180 cells were evaluated by three independent experiments. Values are mean \pm S.E. $(n=3)$

D Box and whisker plots show relative area of mitochondrial membranes to cell area. $* *: p=0.0019, * * * *: p<0.0001, p$ values were obtained from the unpaired two-tailed t-test with Welch's correction.

E mmm1-1 and its corresponding wild-type cells were spotted onto YPD with or without $\mathrm{Tm}$ and cultivated at $23^{\circ} \mathrm{C}$ for 6 days.

F A schematic showing a model that combination of canonical and non-canonical UPR response pathways cooperatively contribute to membrane expansion of the ER and mitochondria to cope with ER stress. 
A
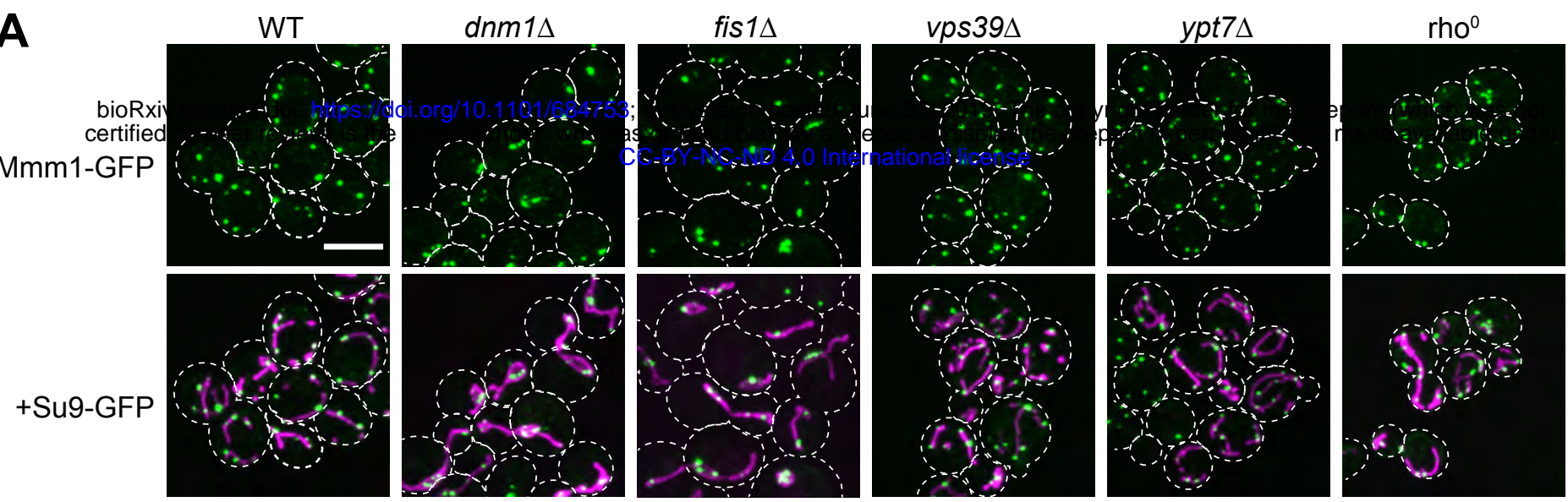

fzo1s

$f z o 1 \Delta d n m 1 \Delta$
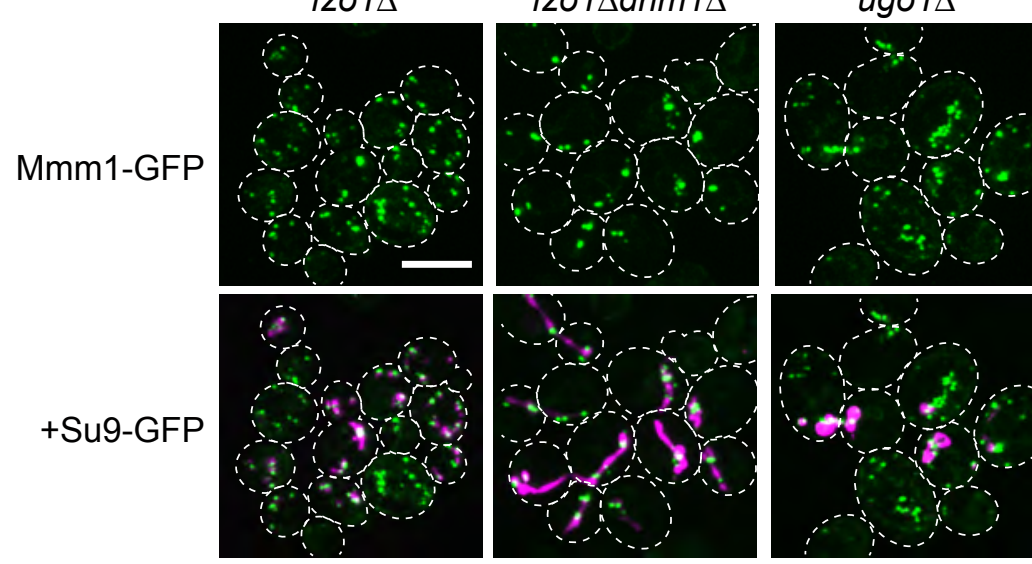

ugo1 $1 \Delta d n m 1 \Delta$
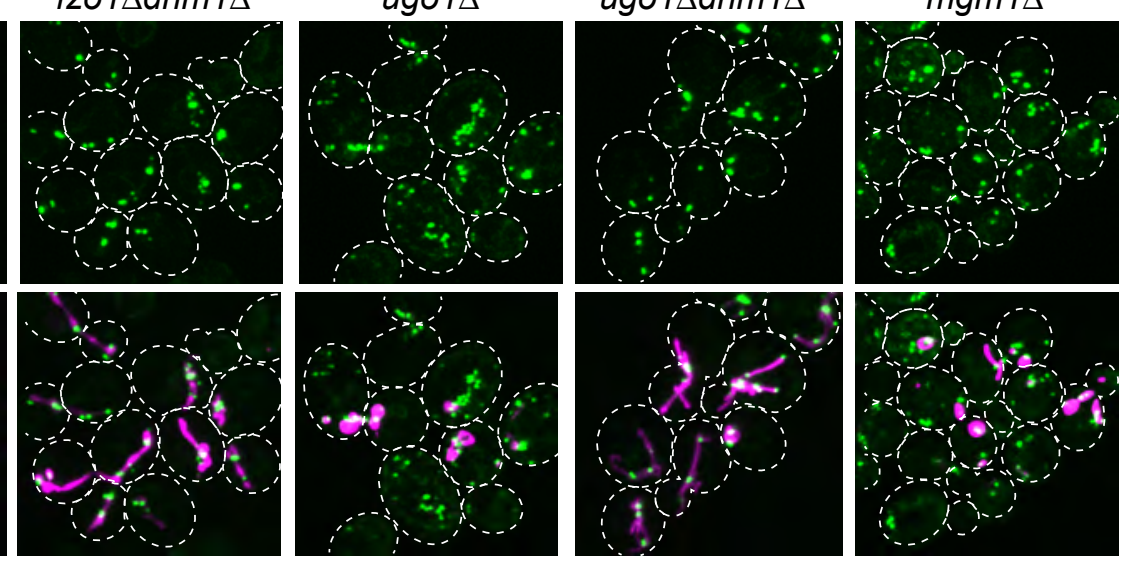

$m g m 1 \Delta d n m 1 \Delta$

B

The number of ERMES foci

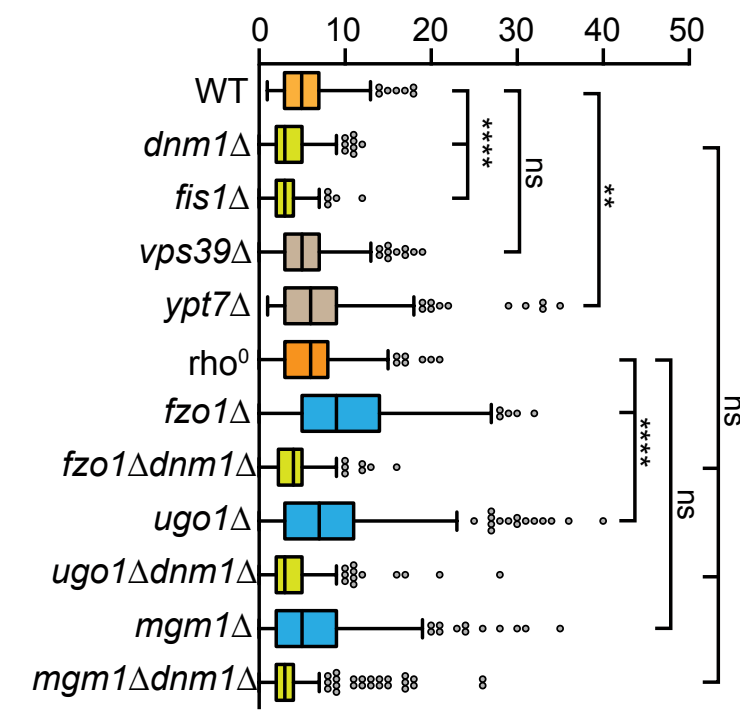

Average $\pm S D$

$5.8 \pm 3.6, n=321$

$3.5 \pm 2.2, n=331$

$3.0 \pm 1.7, n=324$

$5.2 \pm 3.1, n=354$

$6.7 \pm 5.0, n=359$

$6.3 \pm 3.8, n=333$

$10.2 \pm 6.9, n=395$

$3.9 \pm 2.3, n=312$

$8.6 \pm 8.1, n=289$

$3.7 \pm 2.9, n=351$

$6.8 \pm 6.0, n=288$

$3.7 \pm 3.3, n=381$

C Fluorescent Intensity

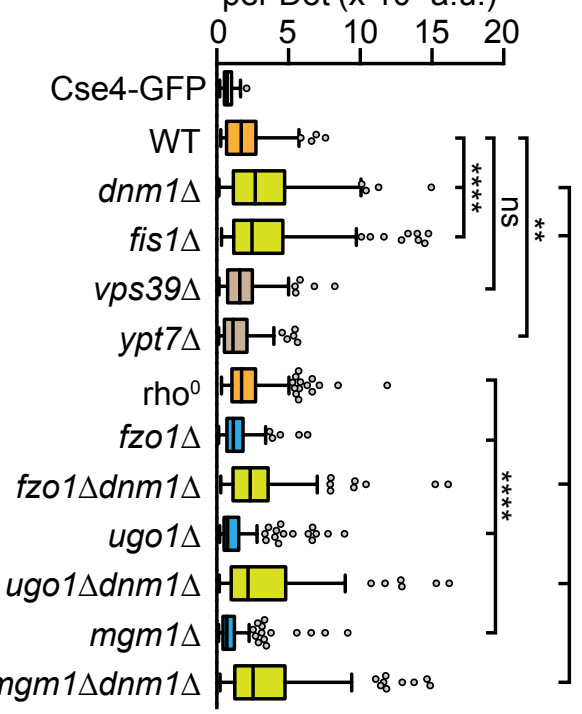

Average \pm SD

$80.0 \pm 2.82, \mathrm{n}=158$

$196 \pm 11.9, n=148$

$335 \pm 22.8, n=140$

$351 \pm 26.3, n=149$

$188 \pm 11.6, n=155$

$148 \pm 9.64, n=153$

$232 \pm 14.9, n=151$

क $143 \pm 8.11, \mathrm{n}=156$

$292 \pm 22.8, n=133$

$138 \pm 12.4, n=154$

$331 \pm 26.8, n=137$

$111 \pm 10.1, n=155$

$356 \pm 27.9, n=135$ 
A

bioRxiv preprint doi: https://doi.org/10.1101/684753; this version posted June 27, 2019. The copyright holder for this preprint (which was not certified by peer review) is the author/funder, who has granted bioRxiva license to display the preprint in perpetuity. It is made available under

GAL1-MMM1- GAL1-MMM1-

aCC-BY-NC-ND 4.Biternational license.

GFP (MATa) mScarlet $(M A T \alpha)$

Haploid
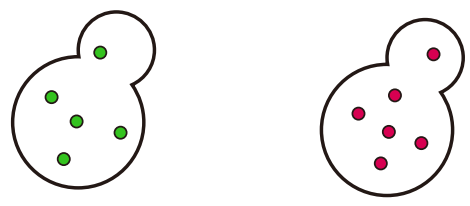

cultivated separately in YPGal

cultivated separately in YPD for 4 hrs

$\checkmark$ to shut off the Mmm1 expression

$\downarrow$ cultivated together for mating
(1)

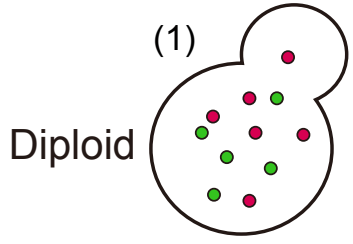

(2)
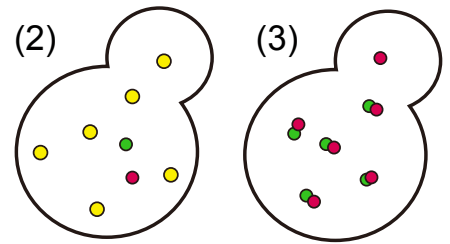

C
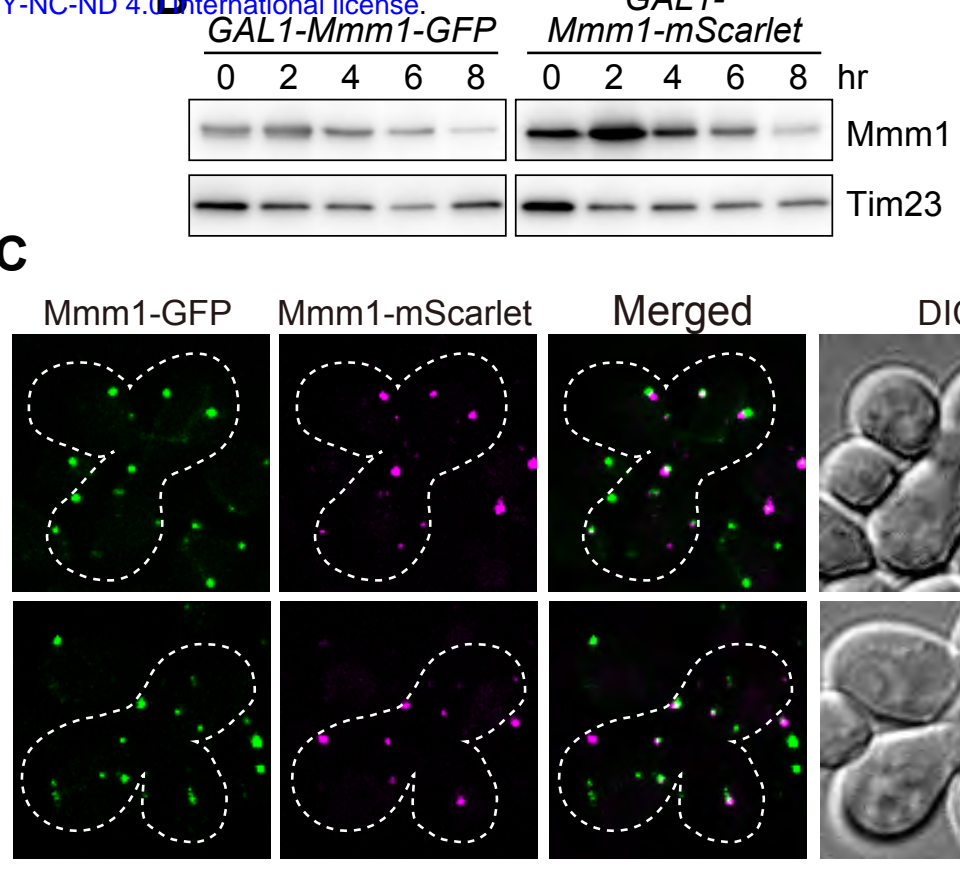

DIC

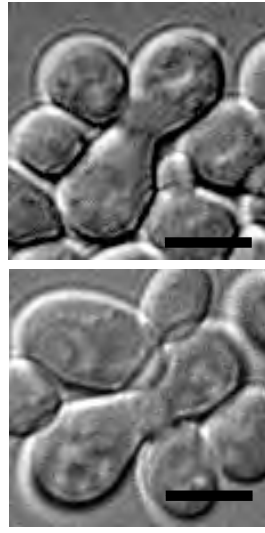

D WT

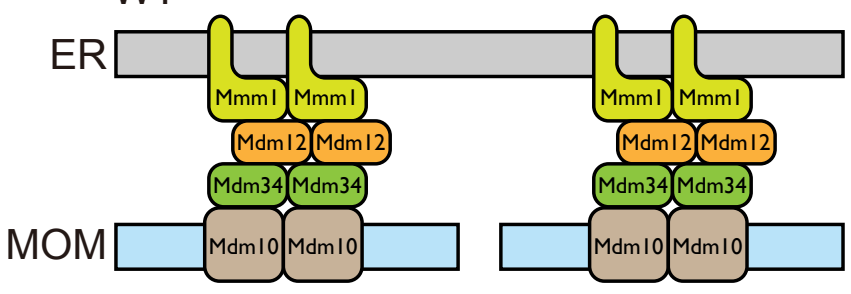

fusions

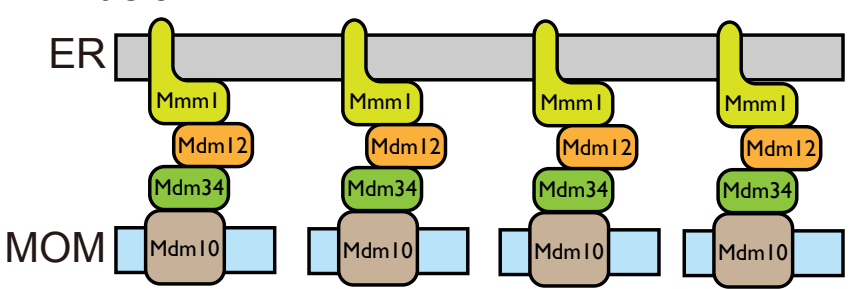

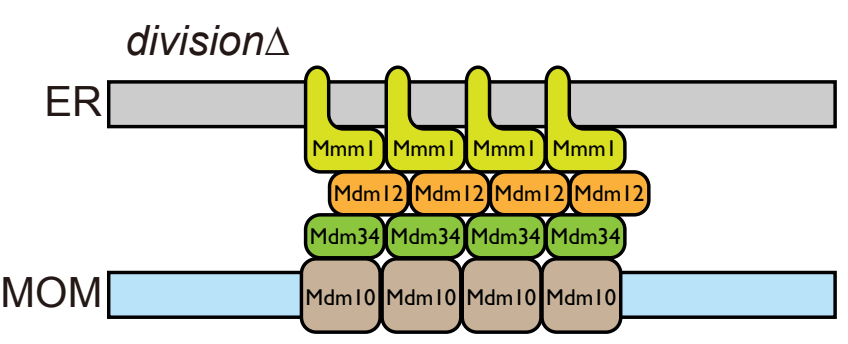

fusions

divisions

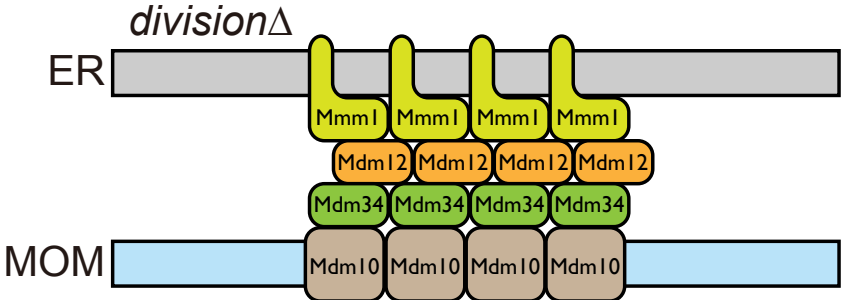




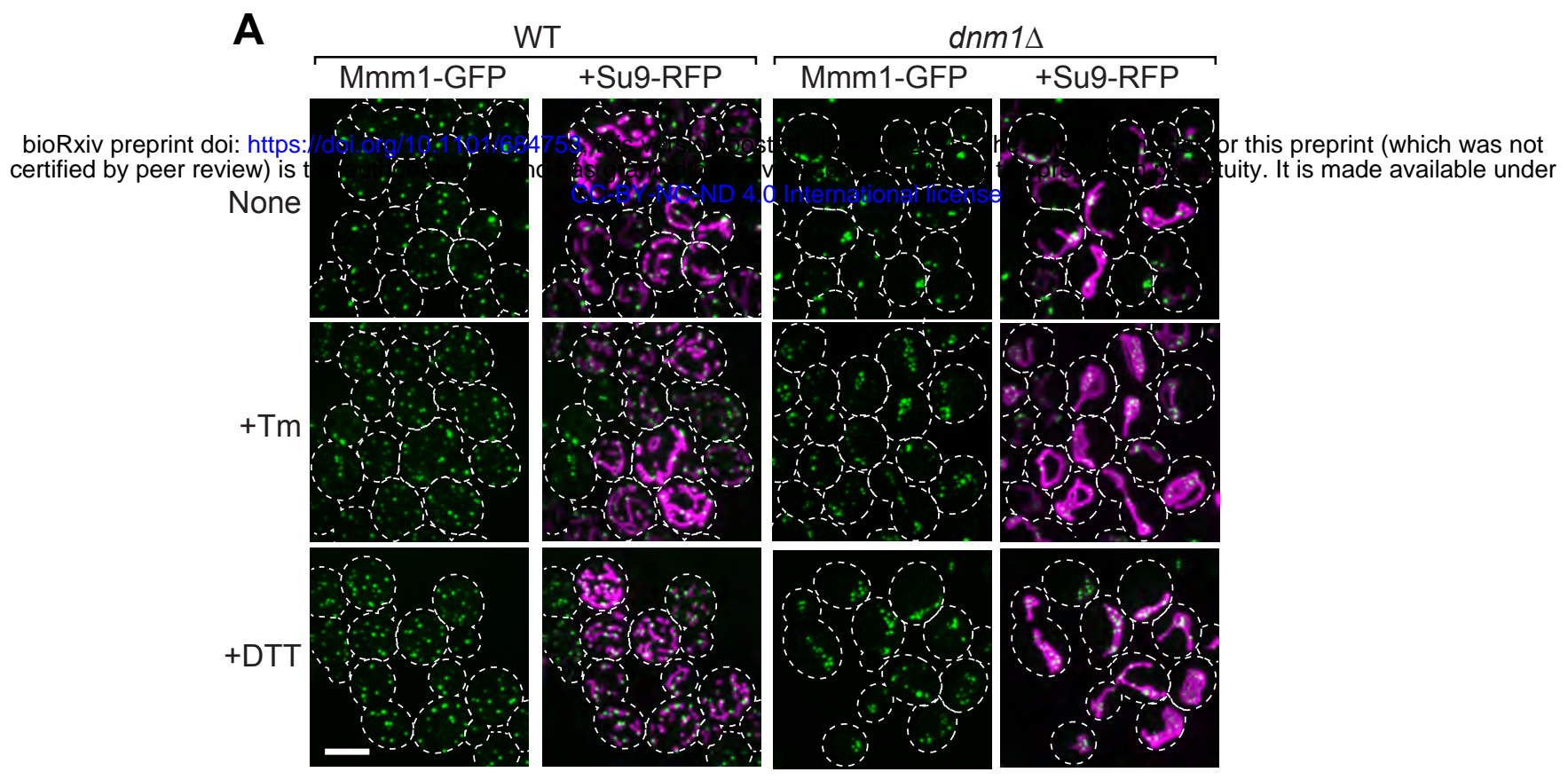

B
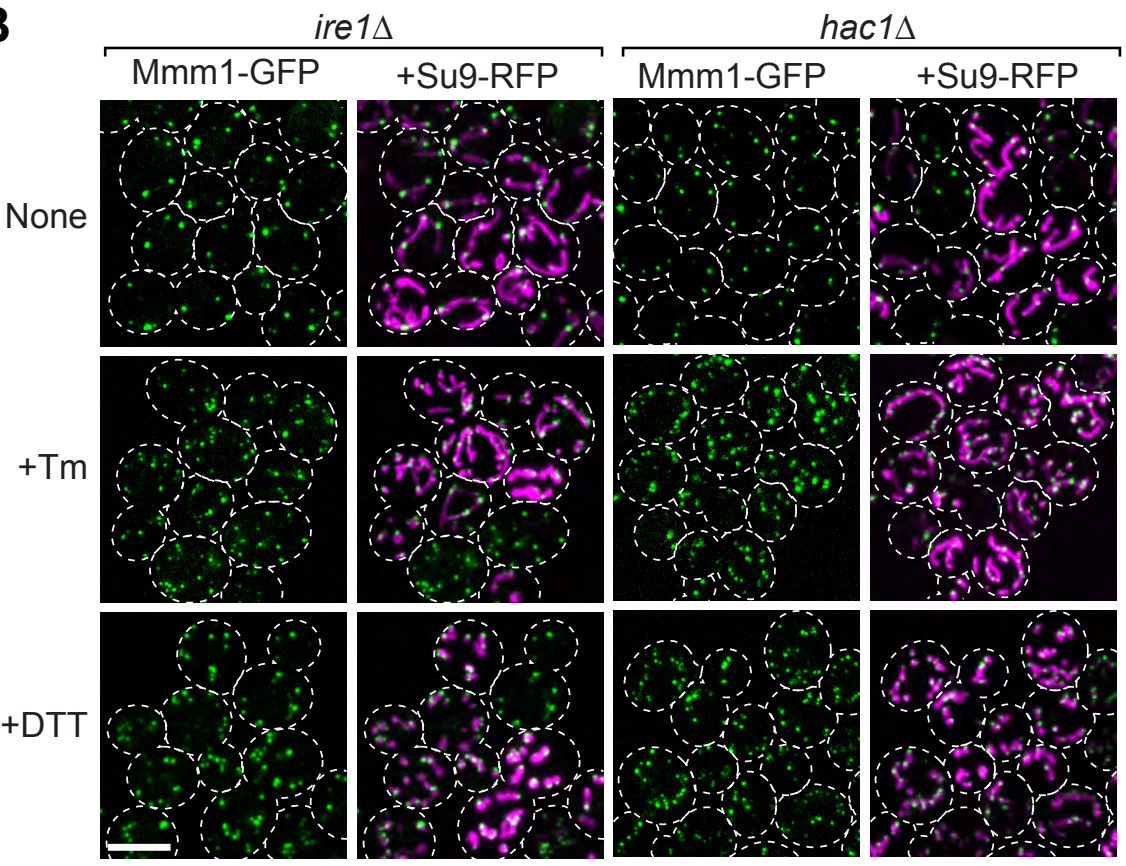

C

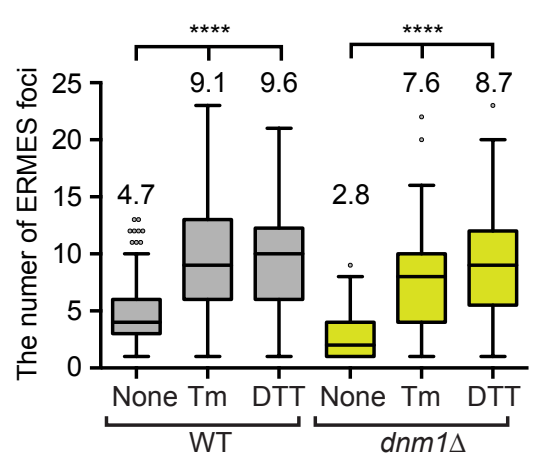

E

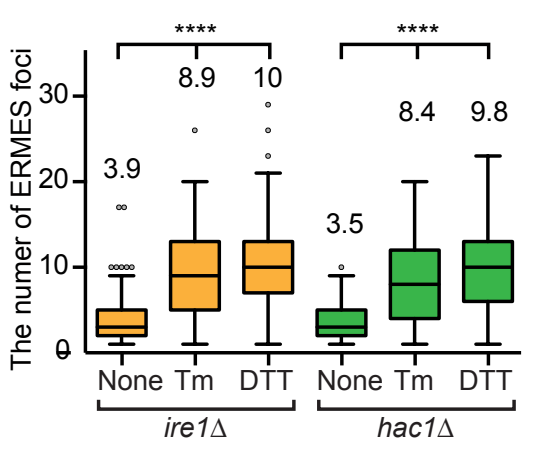

D
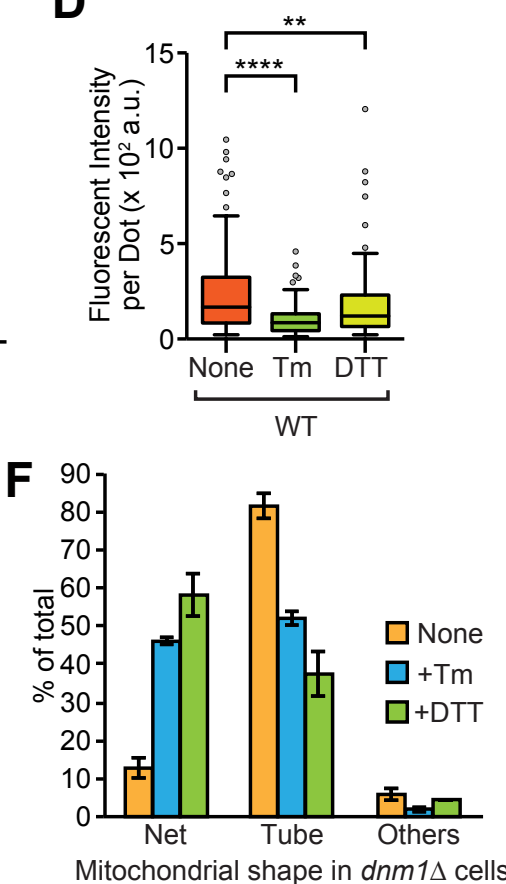


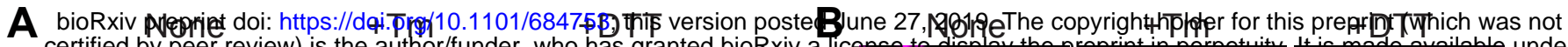
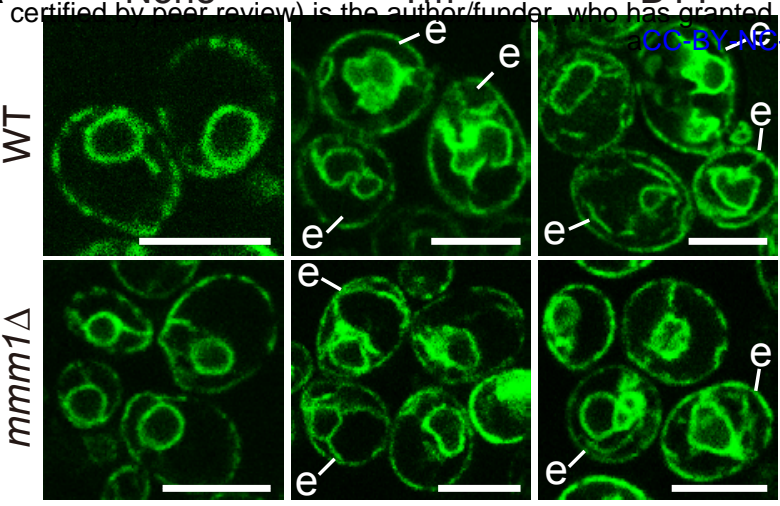

C
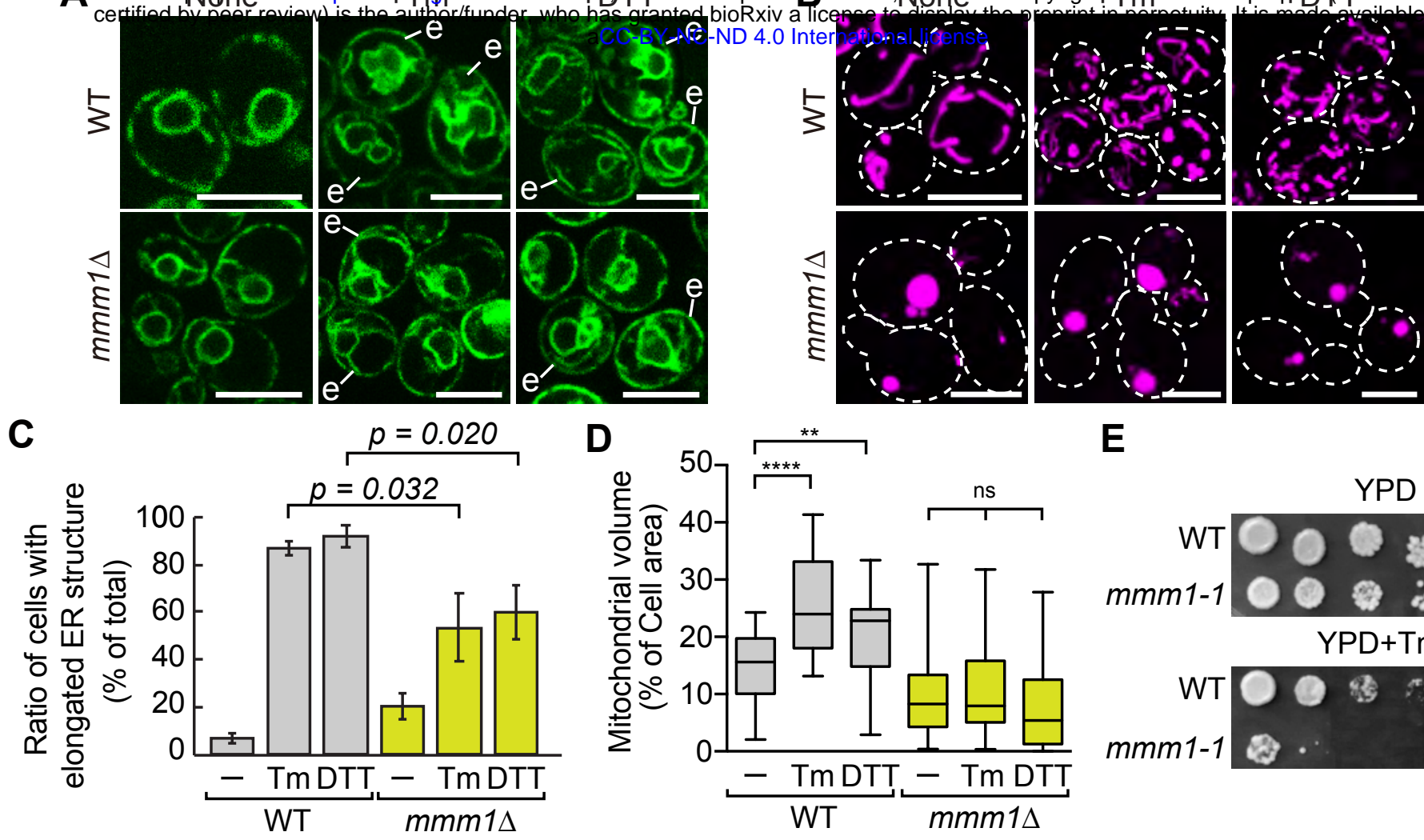

E

$\mathbf{F}$

Connected mitochondria

WT cells

Fragmented

$\left(\begin{array}{cc}0 \\ 0 & 0 \\ 0 & 0\end{array}\right.$

Small number of large ERMES dos
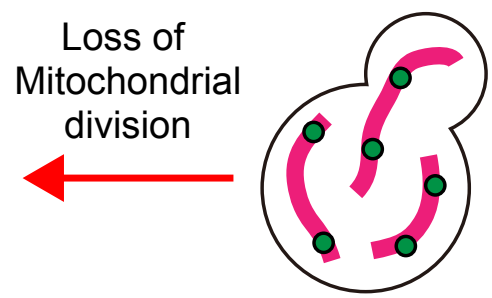

Loss of Mitochondrial mitochondria
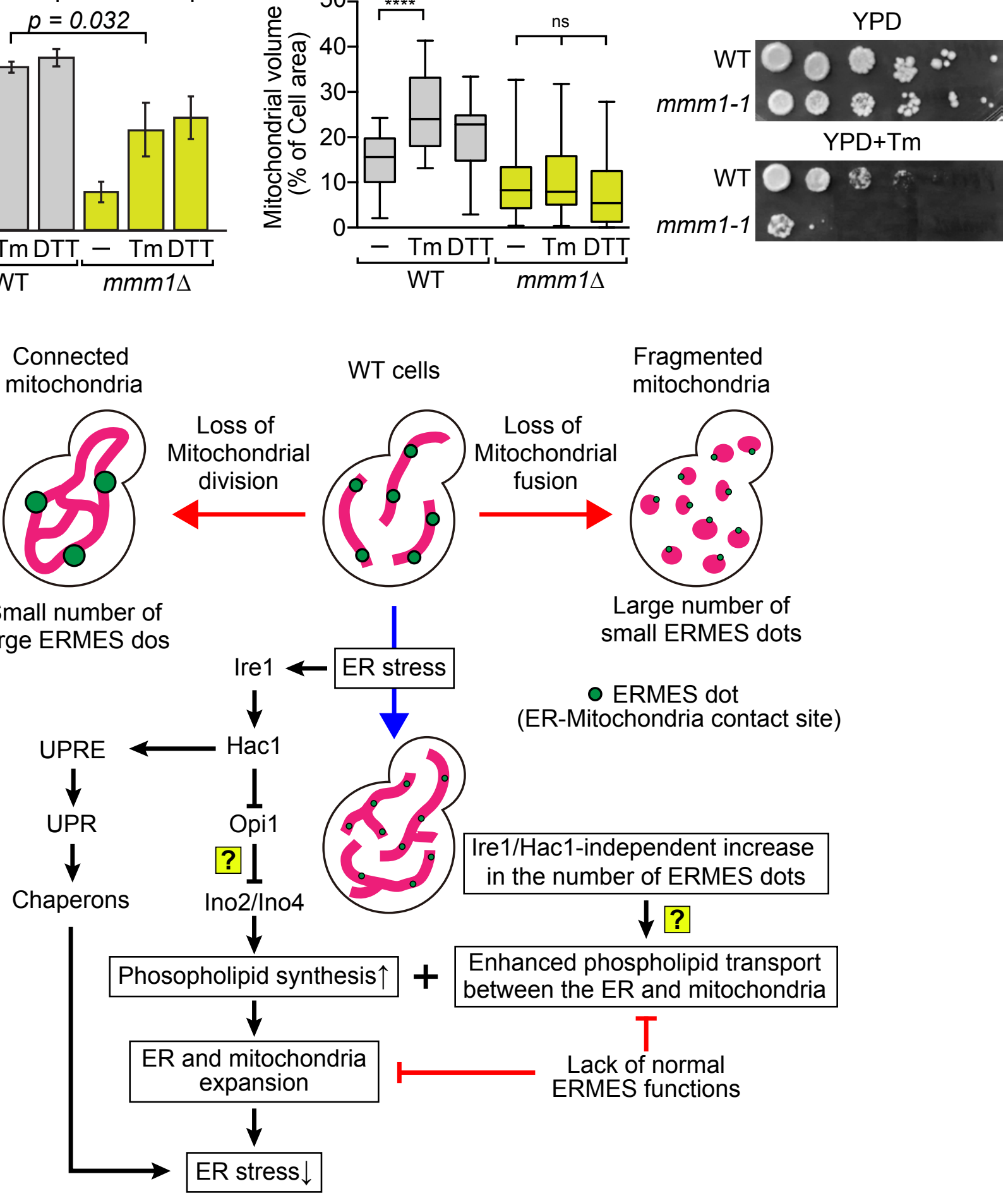